\title{
Relationships between Smoking Status, Cardiovascular Risk Factors, and Lipoproteins in a Large Japanese Population
}

\author{
Marie Nakamura ${ }^{1}$, Yasushi Yamamoto², Wataru Imaoka ${ }^{2}$, Toshio Kuroshima ${ }^{3}$, Ryoko Toragai ${ }^{3}$, Yasuki Ito ${ }^{4}$, \\ Eiichiro Kanda ${ }^{5}$, Ernst $\mathrm{J}$ Schaefer ${ }^{6}$ and Masumi $\mathrm{Ai}^{1}$
}

${ }^{1}$ Department of Insured Medical Care Management, Graduate School of Medical and Dental Sciences, Tokyo Medical and Dental University (TMDU), Tokyo, Japan

${ }^{2}$ Kansai Occupational Health Association, ACTY Health Evaluation Center, Osaka, Japan

${ }^{3}$ Kansai Occupational Health Association, Senri LC Health Evaluation Center, Osaka, Japan

${ }^{4}$ Reagent R\&D department, Research and development Division, Denka Co., Ltd, Niigata, Japan

${ }^{5}$ Medical Science, Kawasaki Medical School, Okayama, Japan

${ }^{6}$ Cardiovascular Nutrition Laboratory, Human Nutrition Research Center on Aging at Tufts University, and Tufts University School of Medicine, MA, Boston, USA

Aims: Smoking is a major risk factor for cardiovascular disease (CVD), a leading cause of death and disability. Other CVD risk factors include age, gender, hypertension, diabetes, increased low-density lipoprotein cholesterol (LDL-C) and decreased high-density lipoprotein cholesterol (HDL-C). Our goal was to assess relationships between smoking status and CVD risk factors, with a focus on direct LDL-C, HDL-C, triglycerides (TG) and small dense LDL-C (sdLDL-C).

Methods: A total of 34,497 Japanese men and women, mean age 51 years, had their CVD risk factors including fasting serum total cholesterol, TG, HDL-C, sdLDL-C, and direct LDL-C assessed. One-way ANOVA and multiple linear regression analyses were carried to assess the interrelationships of these parameters with smoking.

Results: In both men and women, current smokers had significantly $(p<0.001)$ higher median TG $(+19.6 \%$, $+16.9 \%)$ and sdLDL-C levels $(+12.7 \%,+4.2 \%)$ levels, and significantly $(p<0.001)$ lower HDL-C levels $(-7.3 \%$, $-4.3 \%)$ than non-smokers. They were also significantly $(p<0.05)$ more likely to have TG values $>150 \mathrm{mg} / \mathrm{dL}$ $(+56.8 \%,+116.3 \%)$, sdLDL-C $>40.1 \mathrm{mg} / \mathrm{dL}(+28.8 \%,+44.9 \%)$, and HDL-C $<40 \mathrm{mg} / \mathrm{dL}(+89.8 \%,+114.3 \%)$. Ex-smokers generally had lipid values that were intermediate between non-smokers and current smokers. Multivariate analysis confirmed the significance of these relationships.

Conclusion: Our data indicate that current cigarette smoking is associated with increased TG and sdLDL-C levels, as well as decreased HDL-C levels. Furthermore, smoking effect on lipid profiles remain after cessation. These data provide further justification for smoking cessation.

Key words: Smoking, Smoking cessation, Dyslipidemia, Small dense LDL-C, Cardiovascular disease risk

\section{Introduction}

According to the World Health Organization ${ }^{1)}$, cigarette smoking is the single most important preventable cause of morbidity and mortality. Smoking is a highly significant risk factor for lung cancer, chronic obstructive pulmonary disease, and cardiovascular disease (CVD). The Japanese Atherosclerosis Society includes cigarette smoking, along with age, gender, blood pressure, diabetes, low-density lipoprotein cholesterol (LDL-C), high-density lipoprotein cholesterol (HDL-C) and family history of CVD for its CVD risk

Address for correspondence: Masumi Ai, Department of Insured Medical Care Management, Graduate School of Medical and Dental Sciences, Tokyo Medical and Dental University (TMDU), 1-5-45 Yushima, Bunkyo-ku, Tokyo 113-8519, Japan E-mail: ai.vasc@tmd.ac.jp

Received: March 30, 2020 Accepted for publication: September 15, 2020 


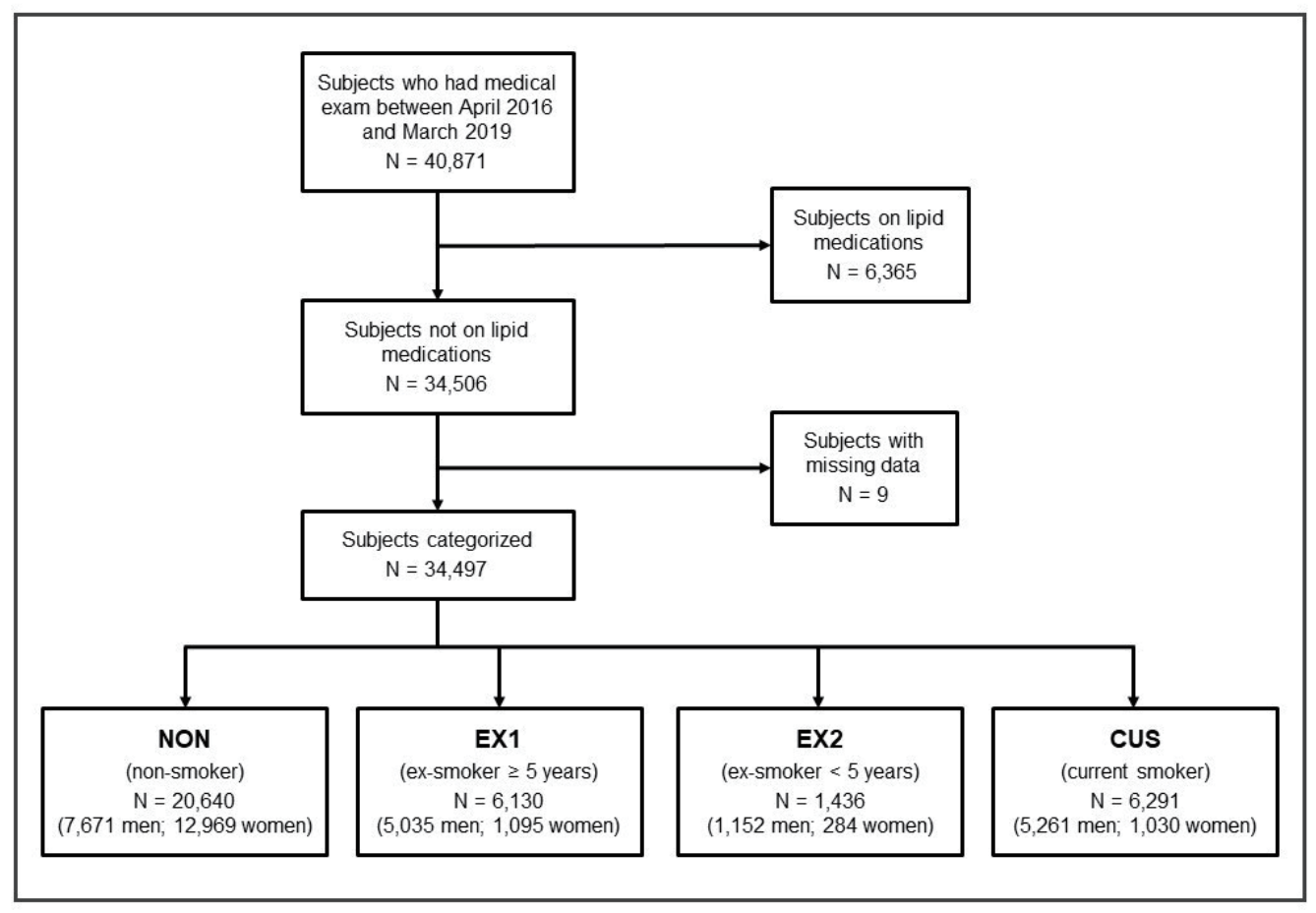

Fig. 1. Flow diagram of subject selection and categorization

prediction algorithm ${ }^{2)}$. A recent report has shown that current smoking was chosen as a parameter in a cardiovascular disease risk prediction model using Suita study ${ }^{3)}$.

There is consensus on the effects of current smoking on CVD but the effects of smoking cessation on CVD were poorly reported. A review suggests the CVD risk caused by smoking is reduced by about half after 4 to 5 years of smoking abstinence ${ }^{4)}$. Based on this review, the American Heart Association and American College of Cardiology reported the estimated risk ratio for atherosclerosis CVD events at 1 to 4 years after smoking cessation ${ }^{5)}$. The estimates for risk reduction associated with smoking cessation is $47 \%$ at 4 years. Furthermore, another study reported that the risk for CVD of former heavy smokers was significantly lower within 5 years relative to current smokers but remained significantly elevated for 5 to 25 years after cessation relative to never smokers ${ }^{6}$. Nevertheless, little is known the mechanism by which the effects after smoking cessation remains.

Anti-inflammatory interventions with anti-cytokine therapy have shown efficacy on reducing cardiovascular events ${ }^{7}$. Smoking contributes CVD through its effects on inflammatory and immune mechanisms. The effects of cigarette smoking on atherosclerosis and CVD risk have mainly focused on its relationship to oxidative stress and endothelial cell dysfunction.
Smoking triggers vascular inflammation by cytokine release, adhesion of inflammatory cells and disruption of endothelial integrity ${ }^{8}$. Also, smoking induces vascular dysfunction by reducing nitric oxide ${ }^{9)}$.

Smoking also contributes to CVD risk via alterations in the lipid profile. It has been reported that smoking lowers HDL-C concentration and elevates triglycerides $(\mathrm{TG})^{10}{ }^{11}$. Additionally, HDL-C was reported to be increased in ex-smokers as compared to current smokers ${ }^{12)}$. On the other hand, there is uncertainty regarding the effects of smoking on LDL-C ${ }^{11)}$. A cross-sectional study showed that mean LDL particle size of current smokers were smaller than those of ex-smokers and non-smokers ${ }^{13)}$. However, another study showed that small dense LDL-C (sdLDL-C) measured by ultracentrifugation method did not correlate with smoking ${ }^{14)}$. At present, there is no clear consensus about association between smoking and serum levels of lipid and lipoprotein subfractions, especially sdLDL-C.

\section{Aim}

Our goal in this investigation was to examine the relationships of current and prior cigarette smoking on LDL-C, HDL-C, TG, and sdLDL-C and other CVD risk factors, using data from a large Japanese population study. 


\section{Methods}

Subjects

Our study population consisted of 40,871 subjects who received a medical examination at the health evaluation centers in Osaka, Japan, between April of 2016 and March of 2019. All subjects agreed to participate in this study and the study protocol had previously been approved by the Medical Research Ethics Committee of Tokyo Medical and Dental University, Tokyo, Japan (M2000-2209). In subjects who had more than one visit during the study period, data on only the first visit was used for this study. Subjects on lipid lowering medication as well as those with missing data were excluded from the analysis (Fig. 1). As CVD risk was reported to be reduced by about half after 4 to 5 years smoking cessation ${ }^{4)}$, subjects were classified into four groups, non-smokers (NON), exsmokers who had quit smoking for $\geq 5$ years from the time of the examination (EX1), ex-smokers who had quit smoking for $<5$ years (EX2), and current smokers (CUS) according to self-reported results on a questionnaire.

\section{Measurements}

Blood samples were collected from all subjects after an overnight fast. Serum creatinine, liver transaminases, total cholesterol (TC), TG, direct LDL-C, and HDL-C were measured by automated standardized enzymatic analysis and sdLDL-C was measured using FDA cleared assay kit (Denka Co. Ltd.) on TBX-FX8 instruments (Canon Medical Systems, Japan) in the health evaluation centers ${ }^{15-21)}$. Serum glucose was measured using the hexokinase G-6-PDH method (FUJIFILM Wako Pure Chemical Co. Japan), and $\mathrm{HbA} 1 \mathrm{c}$ was measured using high performance liquid chromatography on HL-723 G9 instruments (Tosoh, Japan). All assays had within and between run coefficients of variation of $<5.0 \%$. Calculated LDL-C was derived using the Friedewald formula by subtracting the sum of HDL-C and TG/5 from TC ${ }^{22}$. NonHDL-C was calculated by subtracting HDL-C from TC.

\section{Definitions of Hypertension and Diabetes}

We used the blood pressure categories defined by Japanese Society of Hypertension 2019 ${ }^{23)}$. "Hypertension" in Table 2 was defined as using anti-hypertensive drugs or with Stage 1, 2, or 3 hypertension.

We categorized using fasting plasma glucose (FPG) subjects into three groups (FPG $<100 \mathrm{mg} / \mathrm{dl}$; FPG 100-125 mg/dL; and FPG > $125 \mathrm{mg} / \mathrm{dL}$ ). "Diabetes" in Table 2 was defined as having an FPG level $>125 \mathrm{mg} / \mathrm{dL}$ or using anti-diabetic medication.
HbA1c was also used as a marker of diabetes and was reported in Table 1.

\section{Statistical Analysis}

Data are presented as median values with interquartile ranges (IQR) since some variables were not normally distributed. Sd LDL-C and TG data were analyzed after log-transformation of values since these parameters are not normally distributed. Categorical variables are reported as frequencies and percentages. Analyses were performed by gender because of significant differences between men and women with regard to lipid and lipoprotein levels. Univariate analyses were performed by one-way ANOVA for continuous variables. When a significant group effect was observed, the Tukey post-hoc analysis for equally distributed variables and the Games-Howell post-hoc analysis for non-equally distributed variables were performed. Mantel-Haenszel test for trend was used for categorical variables. The 75th percentile of serum sdLDL-C level in this cohort was $40.1 \mathrm{mg} / \mathrm{dL}$, which we used as the cut point. Multivariate linear regression models adjusted for baseline characteristics, such as age and other CVD risk factors were performed to examine the effect of smoking status on lipid profiles. Smoking status was analyzed after converting 3 categorical variables. Values of body mass index (BMI), systolic blood pressure, $\mathrm{HbA1c}$, and drinking habits known as CVD risk factors were chosen as independent variables. No variables showed strong correlation with each other. Akaike's information criteria (AIC) were indicated as fit index. All statistical analyses were performed using SPSS software, version 23 (IBM, Armonk, NY). A $P$ value of $<0.05$ was considered to confer statistical significance in ANOVA, MantelHaenszel test for trend, and multiple regression analysis, while a $P$ value of $<0.001$ was considered statistically significant in the post-hoc analysis in Table 1 , since many comparisons were needed.

\section{Results}

Data on the clinical and laboratory parameters in men $(n=19,119)$ and woman $(n=15,378)$ are shown in Table 1, 2, respectively. EX1 men were significantly older than other groups. However, NON women were the oldest group. The percentage of subjects with obesity (BMI $>25 \mathrm{~kg} / \mathrm{m}^{2}$ ) was higher in EX2 than in other groups. Frequencies of drinking alcoholic beverages were significantly lower in NON subjects than in other groups. In men those that had $\geq 3$ alcoholic drinks/week was $44.4 \%$ in NON and $58.8 \%$ in CUS $(+32.4 \%)$, and in women these values were $22.6 \%$ versus $45.1 \%(+99.6 \%)$, all significantly different. In 
Table 1. Characteristics of subjects by smoking category

\begin{tabular}{|c|c|c|c|c|c|c|c|c|c|c|c|}
\hline \multicolumn{12}{|l|}{ (A) Male Subjects } \\
\hline & & & & & & \multicolumn{6}{|c|}{ PValue ${ }^{\dagger}$} \\
\hline & $\begin{array}{c}\text { Non- } \\
\text { Smokers } \\
(n=7,671)\end{array}$ & $\begin{array}{c}\text { EX1 } \\
\text { Smokers } \\
(n=5,035)\end{array}$ & $\begin{array}{c}\text { EX2 } \\
\text { Smokers } \\
(n=1,152)\end{array}$ & $\begin{array}{c}\text { Current } \\
\text { Smokers } \\
(n=5,261)\end{array}$ & $\begin{array}{c}\text { ANOVA } \\
P \\
\text { Value }^{\S}\end{array}$ & $\begin{array}{c}\text { NON } \\
\text { vs } \\
\text { EX1 }\end{array}$ & $\begin{array}{l}\text { NON } \\
\text { vs } \\
\text { EX2 }\end{array}$ & $\begin{array}{c}\text { NON } \\
\text { vs } \\
\text { CUS }\end{array}$ & $\begin{array}{c}\text { EX1 } \\
\text { vs } \\
\text { EX2 }\end{array}$ & $\begin{array}{c}\text { EX1 } \\
\text { vs } \\
\text { CUS }\end{array}$ & $\begin{array}{c}\text { EX2 } \\
\text { vs } \\
\text { CUS }\end{array}$ \\
\hline Age, year & $\begin{array}{r}48.0 \\
(16.0)\end{array}$ & $\begin{array}{r}55.0 \\
(17.0)\end{array}$ & $\begin{array}{r}47.0 \\
(15.0)\end{array}$ & $\begin{array}{r}48.0 \\
(13.0)\end{array}$ & $<0.001$ & $<0.001$ & 0.058 & $<0.001$ & $<0.001$ & $<0.001$ & 0.952 \\
\hline BMI, $\mathrm{kg} / \mathrm{m}^{2}$ & $\begin{array}{l}23.2 \\
(3.9)\end{array}$ & $\begin{array}{l}23.4 \\
(3.5)\end{array}$ & $\begin{array}{l}23.5 \\
(4.0)\end{array}$ & $\begin{array}{l}23.5 \\
(4.1)\end{array}$ & $<0.001$ & 0.563 & $<0.001$ & 0.007 & 0.005 & 0.216 & 0.139 \\
\hline Systolic blood pressure, $\mathrm{mmHg}$ & $\begin{array}{l}115.0 \\
(21.0)\end{array}$ & $\begin{array}{l}118.0 \\
(22.0)\end{array}$ & $\begin{array}{l}116.0 \\
(21.0)\end{array}$ & $\begin{array}{l}114.0 \\
(21.0)\end{array}$ & $<0.001$ & $<0.001$ & 0.854 & 0.004 & 0.006 & $<0.001$ & 0.041 \\
\hline Diastolic blood pressure, $\mathrm{mmHg}$ & $\begin{array}{r}71.0 \\
(15.0)\end{array}$ & $\begin{array}{r}73.0 \\
(15.0)\end{array}$ & $\begin{array}{r}71.0 \\
(15.8)\end{array}$ & $\begin{array}{r}71.0 \\
(16.0)\end{array}$ & $<0.001$ & $<0.001$ & 0.935 & 0.909 & 0.037 & $<0.001$ & 0.786 \\
\hline \multicolumn{12}{|l|}{ Glucose metabolism } \\
\hline Fasting glucose, mg/dL & $\begin{array}{r}99.0 \\
(11.0)\end{array}$ & $\begin{array}{r}101.0 \\
(13.0)\end{array}$ & $\begin{array}{r}101.0 \\
(13.0)\end{array}$ & $\begin{array}{r}100.0 \\
(13.0)\end{array}$ & $<0.001$ & $<0.001$ & $<0.001$ & $<0.001$ & 0.644 & 0.988 & 0.797 \\
\hline HbAlc, \% & $\begin{array}{r}5.5 \\
(0.4)\end{array}$ & $\begin{array}{r}5.6 \\
(0.4)\end{array}$ & $\begin{array}{r}5.6 \\
(0.4)\end{array}$ & $\begin{array}{r}5.6 \\
(0.4)\end{array}$ & $<0.001$ & $<0.001$ & $<0.001$ & $<0.001$ & 0.419 & 0.007 & 0.988 \\
\hline Albumin, mg/dL & $\begin{array}{r}4.4 \\
(0.3)\end{array}$ & $\begin{array}{r}4.3 \\
(0.4)\end{array}$ & $\begin{array}{r}4.3 \\
(0.3)\end{array}$ & $\begin{array}{r}4.3 \\
(0.3)\end{array}$ & $<0.001$ & $<0.001$ & 0.283 & $<0.001$ & $<0.001$ & 0.002 & 0.004 \\
\hline Creatinine, $\mathrm{mg} / \mathrm{dL}$ & $\begin{array}{r}0.90 \\
(0.16)\end{array}$ & $\begin{array}{r}0.89 \\
(0.17)\end{array}$ & $\begin{array}{r}0.88 \\
(0.14)\end{array}$ & $\begin{array}{r}0.86 \\
(0.16)\end{array}$ & $<0.001$ & 0.498 & 0.002 & $<0.001$ & 0.042 & $<0.001$ & 0.551 \\
\hline ALT, IU/L & $\begin{array}{r}21.0 \\
(14.0)\end{array}$ & $\begin{array}{r}20.0 \\
(13.0)\end{array}$ & $\begin{array}{r}21.0 \\
(16.0)\end{array}$ & $\begin{array}{r}21.0 \\
(16.0)\end{array}$ & $<0.001$ & $<0.001$ & 0.049 & 0.941 & $<0.001$ & $<0.001$ & 0.101 \\
\hline \multicolumn{12}{|l|}{ Lipids } \\
\hline Total cholesterol, mg/dL & $\begin{array}{l}202.0 \\
(42.0)\end{array}$ & $\begin{array}{l}204.0 \\
(40.0)\end{array}$ & $\begin{array}{l}202.0 \\
(44.8)\end{array}$ & $\begin{array}{l}198.0 \\
(45.0)\end{array}$ & $<0.001$ & 0.003 & 0.905 & $<0.001$ & 0.629 & $<0.001$ & 0.001 \\
\hline Triglycerides, $\mathrm{mg} / \mathrm{dL}^{*}$ & $\begin{array}{r}92.0 \\
(67.0)\end{array}$ & $\begin{array}{r}96.0 \\
(68.0)\end{array}$ & $\begin{array}{c}105.0 \\
(77.0)\end{array}$ & $\begin{array}{l}110.0 \\
(84.0)\end{array}$ & $<0.001$ & $<0.001$ & $<0.001$ & $<0.001$ & $<0.001$ & $<0.001$ & 0.374 \\
\hline Direct LDL-C, mg/dL & $\begin{array}{r}122.0 \\
(38.0)\end{array}$ & $\begin{array}{l}121.0 \\
(38.0)\end{array}$ & $\begin{array}{l}122.0 \\
(40.0)\end{array}$ & $\begin{array}{l}120.0 \\
(43.0)\end{array}$ & 0.025 & 0.253 & 0.793 & 0.017 & 0.999 & 0.689 & 0.861 \\
\hline Calculated LDL-C, mg/dL & $\begin{array}{l}123.2 \\
(37.2)\end{array}$ & $\begin{array}{r}122.8 \\
(37.2)\end{array}$ & $\begin{array}{r}122.0 \\
(39.0)\end{array}$ & $\begin{array}{l}118.0 \\
(41.4)\end{array}$ & $<0.001$ & 0.663 & 0.010 & $<0.001$ & 0.070 & $<0.001$ & 0.169 \\
\hline$s \mathrm{dLDL}-\mathrm{C}, \mathrm{mg} / \mathrm{dL}^{*}$ & $\begin{array}{r}30.7 \\
(21.2)\end{array}$ & $\begin{array}{r}33.2 \\
(23.0)\end{array}$ & $\begin{array}{r}35.0 \\
(26.8)\end{array}$ & $\begin{array}{r}34.6 \\
(25.5)\end{array}$ & $<0.001$ & $<0.001$ & $<0.001$ & $<0.001$ & 0.112 & 0.108 & 0.808 \\
\hline sdLDL-C / LDL-C ratio & $\begin{array}{r}0.25 \\
(0.14)\end{array}$ & $\begin{array}{r}0.27 \\
(0.15)\end{array}$ & $\begin{array}{r}0.28 \\
(0.17)\end{array}$ & $\begin{array}{r}0.28 \\
(0.18)\end{array}$ & $<0.001$ & $<0.001$ & $<0.001$ & $<0.001$ & 0.009 & $<0.001$ & 0.999 \\
\hline nonHDL-C, mg/dL & $\begin{array}{l}144.0 \\
(42.0)\end{array}$ & $\begin{array}{l}145.0 \\
(40.0)\end{array}$ & $\begin{array}{l}147.0 \\
(44.0)\end{array}$ & $\begin{array}{l}144.0 \\
(47.0)\end{array}$ & 0.223 & & & & & & \\
\hline HDL-C, mg/dL & $\begin{array}{r}55.0 \\
(18.0)\end{array}$ & $\begin{array}{r}57.0 \\
(19.0)\end{array}$ & $\begin{array}{r}54.0 \\
(16.0)\end{array}$ & $\begin{array}{r}51.0 \\
(17.0)\end{array}$ & $<0.001$ & $<0.001$ & 0.014 & $<0.001$ & $<0.001$ & $<0.001$ & $<0.001$ \\
\hline
\end{tabular}


(Cont. Table 1)

(B) Female Subjects

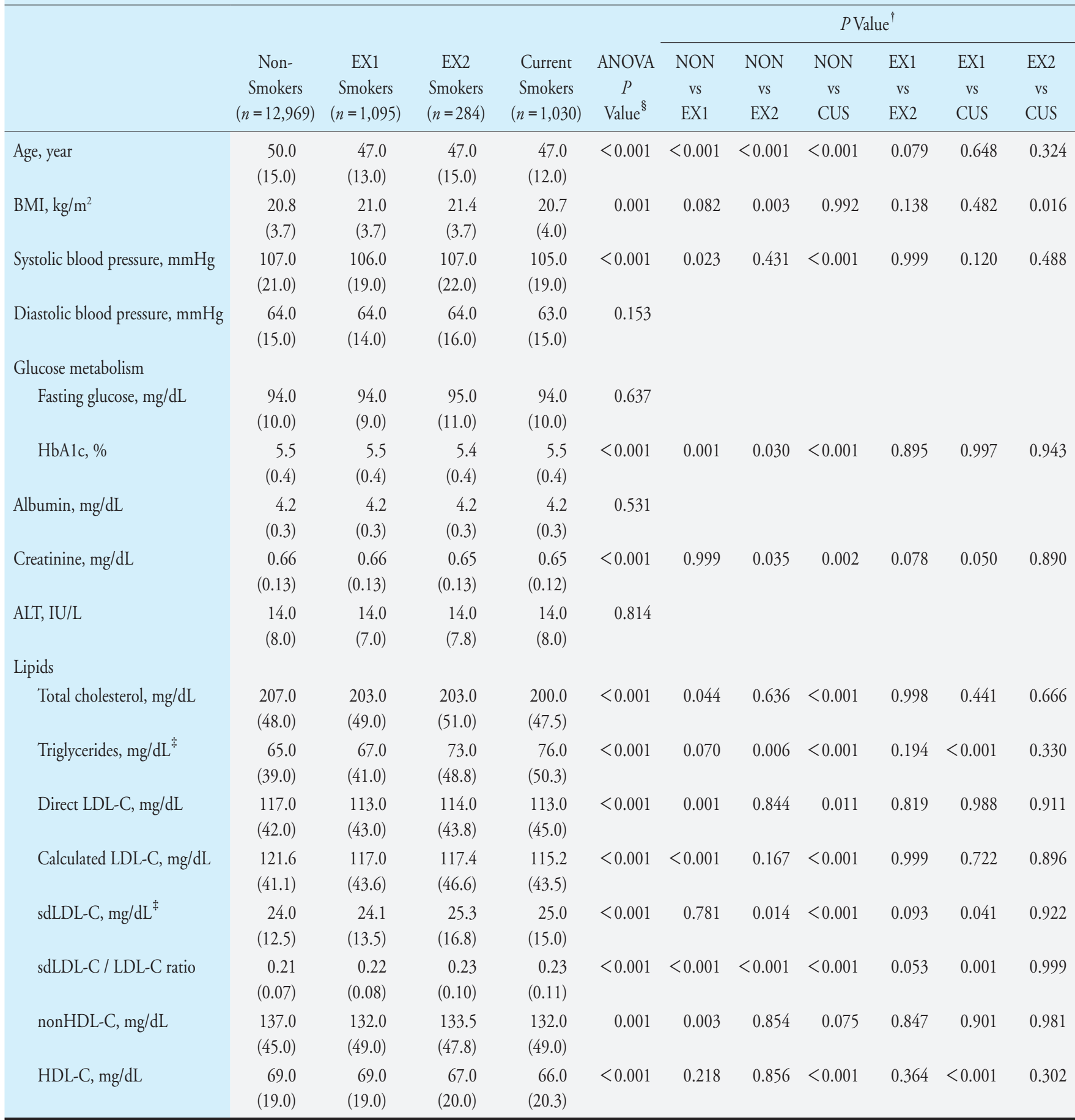

Data are expressed as median (interquartile range).

${ }^{\S} P$ value for comparison across the groups was determined by one-way analysis of variance. $P$ value $<0.05$ was considered statistically significant.

${ }^{\dagger} P$ value for comparison between individual groups, using Tukey post-hoc analysis for equally distributed variables and Games-Howell post-hoc analysis for non-equally distributed variables. $P$ value $<0.001$ was considered statistically significant.

${ }^{*}$ Variable was log-transformed prior to formal statistical analysis.

ALT, alanine aminotransferase; BMI, body mass index; CUS, current smokers; EX1, ex-smokers who had quit smoking for $\geq 5$ years; EX2, exsmokers who had quit smoking for < 5 years; HbA1c, hemoglobin A1c; HDL-C, high-density lipoprotein cholesterol; LDL-C, low-density lipoprotein cholesterol; NON, non-smokers; sdLDL-C, small dense low-density lipoprotein cholesterol 
Table 2. Smoking cohorts stratified by BMI, blood pressure, alcohol consumption, diabetes, and lipid concentrations

\begin{tabular}{|c|c|c|c|c|c|}
\hline \multicolumn{6}{|l|}{ (A) Male Subjects } \\
\hline BMI $>25 \mathrm{~kg} / \mathrm{m}^{2}$ & $2,083(27.2 \%)$ & $1,363(27.1 \%)$ & $372(32.3 \%)$ & $1,574(29.9 \%)$ & $<0.001$ \\
\hline $\mathrm{BMI}>30 \mathrm{~kg} / \mathrm{m}^{2}$ & $330(4.3 \%)$ & $161(3.2 \%)$ & $45(3.9 \%)$ & $227(4.3 \%)$ & 0.782 \\
\hline \multicolumn{6}{|l|}{ Blood pressure, $\mathrm{mmHg}$} \\
\hline Elevated (sBP 130-139 and/or dBP 80-89) & $1,456(19.0 \%)$ & $1,092(21.7 \%)$ & $212(18.4 \%)$ & $889(16.9 \%)$ & \\
\hline Stage 1 HT (sBP 140-159 and/or dBP 90-99) & $674(8.8 \%)$ & $574(11.4 \%)$ & $120(10.4 \%)$ & $466(8.9 \%)$ & \\
\hline Stage 2 HT (sBP 160-179 and/or dBP 100-109) & $152(2.0 \%)$ & $116(2.3 \%)$ & $22(1.9 \%)$ & $104(2.0 \%)$ & \\
\hline Stage $3 \mathrm{HT}(\mathrm{sBP} \geq 180$ and/or dBP $\geq 110)$ & $17(0.2 \%)$ & $14(0.3 \%)$ & $5(0.4 \%)$ & $16(0.3 \%)$ & \\
\hline Hypertension treatment & $914(11.9 \%)$ & $919(18.3 \%)$ & $143(12.4 \%)$ & $525(10.0 \%)$ & $<0.001$ \\
\hline$\geq 3$ drinks per week & $3,407(44.4 \%)$ & $3,297(65.5 \%)$ & $714(62.0 \%)$ & $3,093(58.8 \%)$ & $<0.001$ \\
\hline \multicolumn{6}{|l|}{ Fasting glucose } \\
\hline$<100 \mathrm{mg} / \mathrm{dL}$ & $4,384(57.2 \%)$ & $2,433(48.3 \%)$ & $569(49.4 \%)$ & $2,775(52.7 \%)$ & $<0.001$ \\
\hline $100-125 \mathrm{mg} / \mathrm{dL}$ & $2,922(38.1 \%)$ & $2,269(45.1 \%)$ & $491(42.6 \%)$ & $2,103(40.0 \%)$ & \\
\hline$>125 \mathrm{mg} / \mathrm{dL}$ & $365(4.8 \%)$ & $333(6.6 \%)$ & $92(8.0 \%)$ & $383(7.3 \%)$ & \\
\hline Diabetes treatment & $243(3.2 \%)$ & $214(4.3 \%)$ & $66(5.7 \%)$ & $209(4.0 \%)$ & 0.007 \\
\hline \multicolumn{6}{|l|}{ Diabetes } \\
\hline (fasting glucose $>125 \mathrm{mg} / \mathrm{dL}$ or in-treatment) & $431(5.6 \%)$ & $401(8.0 \%)$ & $106(9.2 \%)$ & $436(8.3 \%)$ & $<0.001$ \\
\hline \multicolumn{6}{|l|}{ Lipids } \\
\hline Triglycerides $>150 \mathrm{mg} / \mathrm{dL}$ & $1,420(18.5 \%)$ & $1,031(20.5 \%)$ & $303(26.3 \%)$ & $1,524(29.0 \%)$ & $<0.001$ \\
\hline
\end{tabular}

(B) Female Subjects

\begin{tabular}{|c|c|c|c|c|c|}
\hline & $\begin{array}{c}\text { Non-Smokers } \\
(n=12,969)\end{array}$ & $\begin{array}{l}\text { EX1 Smokers } \\
(n=1,095)\end{array}$ & $\begin{array}{l}\text { EX2 Smokers } \\
\quad(n=284)\end{array}$ & $\begin{array}{l}\text { Current Smokers } \\
\qquad(n=1,030)\end{array}$ & $\begin{array}{c}P \\
\text { for trend }\end{array}$ \\
\hline $\mathrm{BMI}>25 \mathrm{~kg} / \mathrm{m}^{2}$ & $1,442(11.1 \%)$ & $137(12.5 \%)$ & $44(15.5 \%)$ & $136(13.2 \%)$ & 0.005 \\
\hline $\mathrm{BMI}>30 \mathrm{~kg} / \mathrm{m}^{2}$ & $235(1.8 \%)$ & $23(2.1 \%)$ & $13(4.6 \%)$ & $22(2.1 \%)$ & 0.074 \\
\hline \multicolumn{6}{|l|}{ Blood pressure, $\mathrm{mmHg}$} \\
\hline Normal $(\mathrm{sBP}<120$ and $\mathrm{dBP}<80)$ & $9,616(74.1 \%)$ & $856(78.2 \%)$ & $211(74.3 \%)$ & $826(80.2 \%)$ & \multirow[t]{6}{*}{$<0.001$} \\
\hline Normal high (sBP 120-129 and dBP < 80) & $1,219(9.4 \%)$ & $88(8.0 \%)$ & $30(10.6 \%)$ & $63(6.1 \%)$ & \\
\hline Elevated (sBP 130-139 and/or dBP 80-89) & $1,273(9.8 \%)$ & $97(8.9 \%)$ & $28(9.9 \%)$ & $92(8.9 \%)$ & \\
\hline Stage 1 HT (sBP 140-159 and/or dBP 90-99) & $700(5.4 \%)$ & $44(4.0 \%)$ & $14(4.9 \%)$ & $35(3.4 \%)$ & \\
\hline Stage 2 HT (sBP 160-179 and/or dBP 100-109) & $145(1.1 \%)$ & $9(0.8 \%)$ & $1(0.4 \%)$ & $12(1.2 \%)$ & \\
\hline Stage $3 \mathrm{HT}(\mathrm{sBP} \geq 180$ and/or $\mathrm{dBP} \geq 110)$ & $16(0.1 \%)$ & $1(0.1 \%)$ & $0(0.0 \%)$ & $2(0.1 \%)$ & \\
\hline Hypertension treatment & $844(6.5 \%)$ & $65(5.9 \%)$ & $17(6.0 \%)$ & $56(5.4 \%)$ & 0.136 \\
\hline Hypertension (Stage1-3 HT or in-treatment) & $1,495(11.5 \%)$ & $110(10.0 \%)$ & $32(11.3 \%)$ & $98(9.5 \%)$ & 0.031 \\
\hline \multicolumn{6}{|l|}{ Alcohol consumption } \\
\hline$\geq 1$ drink per week & $4,578(35.3 \%)$ & $592(54.1 \%)$ & $160(56.3 \%)$ & $564(54.8 \%)$ & $<0.001$ \\
\hline$\geq 3$ drinks per week & $2,933(22.6 \%)$ & $450(41.1 \%)$ & $120(42.3 \%)$ & $465(45.1 \%)$ & $<0.001$ \\
\hline \multicolumn{6}{|l|}{ Fasting glucose } \\
\hline$<100 \mathrm{mg} / \mathrm{dL}$ & $10,333(79.7 \%)$ & $872(79.6 \%)$ & $216(76.1 \%)$ & $807(78.3 \%)$ & \multirow[t]{3}{*}{0.168} \\
\hline $100-125 \mathrm{mg} / \mathrm{dL}$ & $2,466(19.0 \%)$ & $209(19.1 \%)$ & $64(22.5 \%)$ & $207(20.1 \%)$ & \\
\hline$>125 \mathrm{mg} / \mathrm{dL}$ & $170(1.3 \%)$ & $14(1.3 \%)$ & $4(1.4 \%)$ & $16(1.6 \%)$ & \\
\hline
\end{tabular}




\begin{tabular}{|c|c|c|c|c|c|}
\hline & $\begin{array}{c}\text { Non-Smokers } \\
(n=12,969)\end{array}$ & $\begin{array}{l}\text { EX1 Smokers } \\
(n=1,095)\end{array}$ & $\begin{array}{l}\text { EX2 Smokers } \\
\quad(n=284)\end{array}$ & $\begin{array}{l}\text { Current Smokers } \\
\qquad(n=1,030)\end{array}$ & $\begin{array}{c}P \\
\text { for trend }\end{array}$ \\
\hline Diabetes treatment & $115(0.9 \%)$ & $4(0.4 \%)$ & $5(1.8 \%)$ & $11(1.1 \%)$ & 0.572 \\
\hline sdLDL-C $>40.1 \mathrm{mg} / \mathrm{dL}$ & $1536(11.8 \%)$ & $148(13.5 \%)$ & $55(19.4 \%)$ & $176(17.1 \%)$ & $<0.001$ \\
\hline $\mathrm{HDL}-\mathrm{C}<40 \mathrm{mg} / \mathrm{dL}$ & $89(0.7 \%)$ & $10(0.9 \%)$ & $0(0.0 \%)$ & $15(1.5 \%)$ & 0.021 \\
\hline
\end{tabular}

men, the prevalence of diabetes was $5.6 \%$ in NON versus $8.3 \%(+48.2 \%)$ in CUS, highly significant; however, such differences were not seen in women (Table 2).

In men, TG levels in the NON group were the lowest of the four groups, and those in the EX1 group were the second lowest, but differences between subjects in the EX2 and CUS group were not significant (Table 1(A)). In women, TG levels in NON and EX1 were similar and lower than those in CUS (Table 1 (B)). Median TG levels were 19.6\% higher in CUS men and $+16.9 \%$ higher in CUS women as compared to NON. In addition, the prevalence of TG levels > $150 \mathrm{mg} / \mathrm{dL}$ was $+56.8 \%$ higher in CUS men at $29.0 \%$ and $116 \%$ higher in CUS women at $10.6 \%$ as compared to $18.5 \%$ and $4.9 \%$ in male and female NON groups (Table 2). These data indicate that smoking is associated with increased TG levels and hypertriglyceridemia; but the increased use of alcoholic drinks in smokers may also have played a role in this association.

In men, sdLDL-C levels in the NON group were significantly lower than those in other groups, with EX1, EX2, and CUS all having similar sdLDL-C levels (Table 1(A)). However, in women, sdLDL-C levels in the NON group were significantly lower than those in the CUS group, while there were no significant difference between EX1, EX2 and other groups (Table 1(B)). Median sdLDL-C were $12.7 \%$ higher in CUS men, but only $4.2 \%$ higher in CUS women as compared to the NON groups (Tables 1 ). In addition, the prevalence of sdLDL-C levels $>40.1 \mathrm{mg} / \mathrm{dL}$ was $28.8 \%$ higher in CUS men at $39.4 \%$, and $44.9 \%$ higher in women at $17.1 \%$, as compared to $30.6 \%$ and $11.8 \%$ in the male and female NON groups (Tables 2). These data indicate that smoking is also associated with increased sdLDL-C levels. In addition, values of sdLDL-C/LDL-C ratio were significantly lower in the NON group than those in other groups both in men and women.

With regard to HDL-C levels, in men these values were highest in the EX1 group. Those in EX2 were significantly lower values than those in the EX1 group, while those in the CUS groups had the lowest values of all the groups (Table $1(\mathrm{~A})$ ). In women, those in the NON and EX1 groups had significantly higher HDL-C levels than those in the CUS group, but there were no significant differences between EX2 and other groups (Table $1(\mathrm{~B})$ ).

Table 3 shows the results of age-adjusted multivariate regression analysis models. After adjustment for age, BMI, sBP, HbA1c, and drinking habits, serum sdLDL-C levels in EX1, EX2 and CUS groups in men and those in EX2 and CUS groups in women were significantly higher than those of the NON group. TG levels in EX1, EX2 and CUS groups were also significantly higher than those in the NON group both in men and women. Analysis using presence of hypertension and diabetes as variables instead of sBP and HbA1c showed same trend (not shown). Moreover, after the adjustment, HDL-C levels in EX2 and CUS groups were significantly lower than those in the NON group in men. Similarly, HDL-C levels in the CUS group were significantly lower than those in the NON group in women. Direct LDL-C levels were not significantly associated with smoking status both in men and women.

\section{Discussion}

Our study shows that smoking status is associated with sdLDL-C, TG and HDL-C levels indepen- 
Table 3. Age-adjusted multiple regression analysis with sdLDL-C, triglycerides, HDL-C and direct LDL-C

\begin{tabular}{|c|c|c|c|c|}
\hline & $\log$ sdLDL-C & $\log$ Triglycerides & HDL-C & direct LDL-C \\
\hline & B $(95 \% \mathrm{CI})$ & B $(95 \%$ CI $)$ & B $(95 \% \mathrm{CI})$ & B $(95 \% \mathrm{CI})$ \\
\hline \multicolumn{5}{|l|}{ Model 1} \\
\hline Non-smokers & Reference & Reference & Reference & Reference \\
\hline EX1 smokers & $0.059(0.042,0.076)$ & $0.051(0.032,0.071)$ & $0.960(0463,1.457)$ & $-0.986(-2.070,0.097)$ \\
\hline EX2 smokers & $0.107(0.078,0.136)$ & $0.162(0.129,0.196)$ & $-1.207(-2.055,-0.358)$ & $-0.856(-2.706,0.993)$ \\
\hline \multirow[t]{2}{*}{ Current smokers } & $0.094(0.077,0.110)$ & $0.192(0.174,0.211)$ & $-3.565(-4.046,-3.084)$ & $-1.623(-2.671,-0.574)$ \\
\hline & $\mathrm{AIC}=-29046.2$ & $\mathrm{AIC}=-23938.4$ & $\mathrm{AIC}=100074.1$ & $\mathrm{AIC}=129881.4$ \\
\hline \multicolumn{5}{|l|}{ Model 2} \\
\hline Non-smokers & Reference & Reference & Reference & Reference \\
\hline EX1 smokers & $0.053(0.037,0.069)$ & $0.044(0.026,0.062)$ & $1.163(0.700,1.626)$ & $-1.223(-2.286,-0.161)$ \\
\hline EX2 smokers & $0.090(0.062,0.118)$ & $0.139(0.108,0.170)$ & $-0.568(-1.359,0.222)$ & $-1.601(-3.416,0.214)$ \\
\hline Current smokers & $0.086(0.070,0.102)$ & $0.182(0.164,0.199)$ & $-3.283(-3.731,-2.835)$ & $-1.951(-2.980,-0.923)$ \\
\hline \multirow[t]{2}{*}{ BMI } & $0.043(0.041,0.045)$ & $0.057(0.055,0.059)$ & $-1.545(-1.601,-1.489)$ & $1.802(1.674,1.931)$ \\
\hline & $\mathrm{AIC}=-30779.4$ & $\mathrm{AIC}=-26367.4$ & $\mathrm{AIC}=97349.2$ & $\mathrm{AIC}=129141.8$ \\
\hline \multicolumn{5}{|l|}{ Model 3} \\
\hline Non-smokers & Reference & Reference & Reference & Reference \\
\hline EX1 smokers & $0.026(0.010,0.042)$ & $0.034(0.016,0.053)$ & $0.016(-0.438,0.470)$ & $0.178(-0.887,1.243)$ \\
\hline EX2 smokers & $0.062(0.035,0.090)$ & $0.129(0.098,0.161)$ & $-1.720(-2.490,-0.950)$ & $-0.194(-2.001,1.613)$ \\
\hline Current smokers & $0.064(0.048,0.079)$ & $0.174(0.156,0.192)$ & $-4.231(-4.670,-3.793)$ & $-0.793(-1.822,0.235)$ \\
\hline $\mathrm{BMI}$ & $0.044(0.042,0.045)$ & $0.057(0.055,0.060)$ & $-1.506(-1.561,-1.452)$ & $1.755(1.628,1.883)$ \\
\hline \multirow[t]{2}{*}{$\geq 3$ drinks per week } & $0.150(0.137,0.163)$ & $0.051(0.036,0.065)$ & $6.302(5.941,6.664)$ & $-7.697(-8.545,-6.848)$ \\
\hline & $\mathrm{AIC}=-31290.2$ & $\mathrm{AIC}=-26411.7$ & $\mathrm{AIC}=96217.4$ & $\mathrm{AIC}=128829.9$ \\
\hline \multicolumn{5}{|l|}{ Model 4} \\
\hline Non-smokers & Reference & Reference & Reference & Reference \\
\hline EX1 smokers & $0.025(0.009,0.041)$ & $0.034(0.016,0.052)$ & $0.023(-0.431,0.476)$ & $0.156(-0.908,1.219)$ \\
\hline EX2 smokers & $0.054(0.026,0.081)$ & $0.122(0.091,0.153)$ & $-1.631(-2.401,-0.860)$ & $-0.486(-2.292,1.320)$ \\
\hline Current smokers & $0.054(0.038,0.069)$ & $0.165(0.148,0.183)$ & $-4.128(-4.568,-3.689)$ & $-1.129(-2.160,-0.098)$ \\
\hline BMI & $0.040(0.038,0.042)$ & $0.054(0.052,0.057)$ & $-1.470(-1.526,-1.415)$ & $1.638(1.507,1.769)$ \\
\hline$\geq 3$ drinks per week & $0.159(0.146,0.172)$ & $0.059(0.044,0.074)$ & $6.207(5.844,6.570)$ & $-7.388(-8.239,-6.537)$ \\
\hline \multirow[t]{2}{*}{$\mathrm{HbAlc}$} & $0.079(0.068,0.090)$ & $0.070(0.058,0.082)$ & $-0.814(-1.113,-0.514)$ & $2.647(1.945,3.349)$ \\
\hline & $\mathrm{AIC}=-31499.8$ & $\mathrm{AIC}=-26539.3$ & $\mathrm{AIC}=96191.0$ & $\mathrm{AIC}=128777.3$ \\
\hline \multicolumn{5}{|l|}{ Model 5} \\
\hline Non-smokers & Reference & Reference & Reference & Reference \\
\hline EX1 smokers & $0.026(0.010,0.042)$ & $0.035(0.016,0.053)$ & $0.036(-0.417,0.489)$ & $0.156(-0.907,1.220)$ \\
\hline EX2 smokers & $0.056(0.029,0.084)$ & $0.124(0.093,0.155)$ & $-1.582(-2.350,-0.813)$ & $-0.483(-2.290,1.323)$ \\
\hline Current smokers & $0.059(0.044,0.075)$ & $0.170(0.152,0.188)$ & $-4.029(-4.468,-3.590)$ & $-1.123(-2.155,-0.092)$ \\
\hline BMI & $0.035(0.033,0.037)$ & $0.050(0.048,0.052)$ & $-1.559(-1.618,-1.501)$ & $1.633(1.495,1.771)$ \\
\hline$\geq 3$ drinks per week & $0.145(0.132,0.158)$ & $0.047(0.032,0.062)$ & $5.963(5.598,6.328)$ & $-7.402(-8.261,-6.543)$ \\
\hline $\mathrm{HbA1c}$ & $0.077(0.066,0.087)$ & $0.068(0.056,0.080)$ & $-0.856(-1.155,-0.557)$ & $2.645(1.943,3.347)$ \\
\hline \multirow[t]{2}{*}{ sBP } & $0.003(0.003,0.004)$ & $0.003(0.002,0.003)$ & $0.058(0.047,0.070)$ & $0.003(-0.025,0.031)$ \\
\hline & $\mathrm{AIC}=-31735.9$ & $\mathrm{AIC}=-26670.6$ & $\mathrm{AIC}=96099.3$ & $\mathrm{AIC}=128779.3$ \\
\hline
\end{tabular}


(Cont. Table 3)

(B) Female Subjects

\begin{tabular}{|c|c|c|c|c|}
\hline & $\log$ sdLDL-C & log Triglycerides & HDL-C & direct LDL-C \\
\hline & B (95\% CI) & B (95\% CI) & B $(95 \% \mathrm{CI})$ & B (95\% CI) \\
\hline \multicolumn{5}{|l|}{ Model 1} \\
\hline Non-smokers & Reference & Reference & Reference & Reference \\
\hline EX1 smokers & $0.036(0.012,0.060)$ & $0.057(0.031,0.084)$ & $1.059(0.143,1.974)$ & $-1.861(-3.653,-0.069)$ \\
\hline EX2 smokers & $0.127(0.082,0.173)$ & $0.145(0.095,0.195)$ & $-0.405(-2.150,1.340)$ & $1.831(-1.585,5.247)$ \\
\hline \multirow[t]{2}{*}{ Current smokers } & $0.093(0.068,0.118)$ & $0.189(0.161,0.216)$ & $-2.241(-3.183,-1.299)$ & $-0.911(-2.756,0.933)$ \\
\hline & $\mathrm{AIC}=-29040.6$ & $\mathrm{AIC}=-26147.6$ & $\mathrm{AIC}=82930.0$ & $\mathrm{AIC}=103599.9$ \\
\hline \multicolumn{5}{|l|}{ Model 2} \\
\hline Non-smokers & Reference & Reference & Reference & Reference \\
\hline EX1 smokers & $0.027(0.004,0.050)$ & $0.045(0.020,0.069)$ & $1.488(0.626,2.351)$ & $-2.474(-4.212,-0.735)$ \\
\hline EX2 smokers & $0.102(0.057,0.146)$ & $0.108(0.061,0.155)$ & $0.843(-0.801,2.487)$ & $0.052(-3.263,3.367)$ \\
\hline Current smokers & $0.090(0.066,0.114)$ & $0.185(0.159,0.210)$ & $-2.109(-2.996,-1.222)$ & $-1.100(-2.888,0.689)$ \\
\hline \multirow[t]{2}{*}{ BMI } & $0.032(0.030,0.034)$ & $0.046(0.044,0.048)$ & $-1.556(-1.625,-1.487)$ & $2.218(2.079,2.357)$ \\
\hline & $\mathrm{AIC}=-30149.2$ & $\mathrm{AIC}=-28091.7$ & $\mathrm{AIC}=81095.7$ & $\mathrm{AIC}=102656.4$ \\
\hline \multicolumn{5}{|l|}{ Model 3} \\
\hline Non-smokers & Reference & Reference & Reference & Reference \\
\hline EX1 smokers & $0.013(-0.010,0.037)$ & $0.046(0.021,0.071)$ & $0.279(-0.572,1.130)$ & $-0.693(-2.424,1.039)$ \\
\hline EX2 smokers & $0.086(0.042,0.131)$ & $0.109(0.062,0.157)$ & $-0.482(-2.098,1.134)$ & $2.003(-1.285,5.291)$ \\
\hline Current smokers & $0.073(0.049,0.097)$ & $0.186(0.160,0.212)$ & $-3.576(-4.454,-2.698)$ & $1.060(-0.726,2.847)$ \\
\hline BMI & $0.033(0.031,0.034)$ & $0.046(0.044,0.048)$ & $-1.522(-1.590,-1.454)$ & $2.168(2.030,2.305)$ \\
\hline \multirow[t]{2}{*}{$\geq 3$ drinks per week } & $0.072(0.059,0.086)$ & $-0.077(-0.022,0.008)$ & $6.351(5.848,6.854)$ & $-9.351(-10.375,-8.327)$ \\
\hline & $\mathrm{AIC}=-30253.8$ & $\mathrm{AIC}=-28090.5$ & $\mathrm{AIC}=80497.5$ & $\mathrm{AIC}=102341.0$ \\
\hline \multicolumn{5}{|l|}{ Model 4} \\
\hline Non-smokers & Reference & Reference & Reference & Reference \\
\hline EX1 smokers & $0.015(-0.008,0.038)$ & $0.047(0.022,0.072)$ & $0.264(-0.587,1.114)$ & $-0.591(-2.311,1.129)$ \\
\hline EX2 smokers & $0.089(0.045,0.133)$ & $0.111(0.064,0.158)$ & $-0.507(-2.123,1.108)$ & $2.172(-1.094,5.438)$ \\
\hline Current smokers & $0.074(0.050,0.097)$ & $0.186(0.161,0.212)$ & $-3.577(-4.455,-2.700)$ & $1.067(-0.707,2.842)$ \\
\hline BMI & $0.029(0.028,0.031)$ & $0.044(0.042,0.046)$ & $-1.492(-1.561,-1.423)$ & $1.970(1.830,2.110)$ \\
\hline$\geq 3$ drinks per week & $0.085(0.072,0.099)$ & $0.003(-0.012,0.018)$ & $6.223(5.717,6.730)$ & $-8.498(-9.522,-7.475)$ \\
\hline \multirow[t]{2}{*}{$\mathrm{HbA1c}$} & $0.134(0.118,0.150)$ & $0.103(0.086,0.121)$ & $-1.314(-1.904,-0.723)$ & $8.764(7.570,9.958)$ \\
\hline & $\mathrm{AIC}=-30518.4$ & $\mathrm{AIC}=-28226.4$ & $\mathrm{AIC}=80480.4$ & $\mathrm{AIC}=102137.4$ \\
\hline \multicolumn{5}{|l|}{ Model 5} \\
\hline Non-smokers & Reference & Reference & Reference & Reference \\
\hline EX1 smokers & $0.019(-0.004,0.042)$ & $0.050(0.026,0.075)$ & $0.293(-0.558,1.144)$ & $-0.510(-2.231,1.120)$ \\
\hline EX2 smokers & $0.093(0.050,0.137)$ & $0.115(0.068,0.162)$ & $-0.476(-2.091,1.139)$ & $2.259(-1.006,5.524)$ \\
\hline Current smokers & $0.081(0.057,0.104)$ & $0.192(0.166,0.217)$ & $-3.526(-4.404,-2.648)$ & $1.210(-0.566,2.985)$ \\
\hline BMI & $0.025(0.023,0.027)$ & $0.040(0.038,0.042)$ & $-1.526(-1.599,-1.453)$ & $1.877(1.729,2.025)$ \\
\hline$\geq 3$ drinks per week & $0.079(0.065,0.092)$ & $-0.002(-0.017,0.013)$ & $6.175(5.668,6.682)$ & $-8.632(-9.658,-7.607)$ \\
\hline HbA1c & $0.133(0.117,0.149)$ & $0.103(0.086,0.120)$ & $-1.321(-1.911,-0.730)$ & $8.746(7.552,9.939)$ \\
\hline \multirow[t]{2}{*}{ sBP } & $0.003(0.002,0.003)$ & $0.002(0.002,0.003)$ & $0.021(0.006,0.035)$ & $0.057(0.028,0.087)$ \\
\hline & $\mathrm{AIC}=-30717.6$ & $\mathrm{AIC}=-28330.9$ & $\mathrm{AIC}=80474.8$ & $\mathrm{AIC}=102125.0$ \\
\hline
\end{tabular}

AIC, Akaike's Information Criterion; BMI, body mass index; EX1, ex-smokers who had quit smoking for $\geq 5$ years; EX2, ex-smokers who had quit smoking for < 5 years; HbA1c, hemoglobin A1c; HDL-C, high-density lipoprotein; LDL-C, low-density lipoprotein; sBP, systolic blood pressure; sdLDL-C, small dense low-density lipoprotein cholesterol 
dently of other CVD risk factors. CUS had significantly higher sdLDL-C and TG levels, and significantly lower HDL-C values than NON. Furthermore, our results also indicate that EX1 had lower TG levels and higher HDL-C levels than CUS. However, the differences of sdLDL-C levels between EX1 and CUS were not significant both in men and women. In men values of sdLDL-C and TG remained higher in EX1 than in the NON group.

Previous studies have shown that cigarette smoking lowers HDL-C levels and increases TG levels, consistent with our data ${ }^{10,11)}$. In addition, one cross-sectional study showed that mean LDL particle size of current smoker were smaller than those of ex-smokers and non-smokers, also consistent with our results ${ }^{13)}$. However, another study showed that sdLDL-C measured after ultracentrifugation did not correlate with smoking status ${ }^{14)}$. This difference could be because of the small number of subjects studied as well as the method of analysis. Our sdLDL-C concentration data was measured by automated FAD-cleared enzymatic analysis so that the measurement error would be expected to be substantially less than for data generated by ultracentrifugation analysis ${ }^{16,17)}$. In a randomized clinical trial, smoking cessation over a one-year period did not change the prevalence of small LDL particles ${ }^{12)}$. In contrast, our study documented that sdLDL-C levels were significantly lower in subjects who had quit smoking for over 5 years as compared than current smokers. However, there was no difference in our analysis between subjects who have quit smoking for less than 5 years and current smokers. These results suggest that the effects of smoking on sdLDL-C may persist for a number of years after smoking cessation, consistent with reports that smoking continues to affect CVD after smoking cessation $^{4-6)}$. Another possible explanation would be that EX2 group might include people who had just quit or had quit for some health reasons, and they might keep unfavorable lipid profiles.

Nicotine and other particles in cigarette are known to promote secretion of catecholamines, cortisol and growth hormones, and as a result, free fatty acids are released, which in turn affects very-low-density lipoprotein (VLDL) and LDL concentrations in blood, contributing to lower HDL concentrations ${ }^{24)}$. It has been reported that lipoprotein lipase activity in skeletal muscle (known to regulate TG levels) is reduced in smokers compared to non-smokers ${ }^{25)}$. SdLDL-C level has been reported to correlate strongly with TG level, and the same association was observed in our study ${ }^{26-28)}$. TG elevation would be a part of the cause of sdLDL-C elevation in current smokers.

In men, TG levels were lower in EX1 than in
CUS, and sdLDL-C levels in EX1 were similar to that in CUS but were still higher than in NON. These data indicate that the effects of smoking may persist for some time after smoking cessation, and therefore cannot be explained by the short-term effects of nicotine and other particles in cigarettes. In our data, participants in CUS, EX1, and EX2 groups had a higher drinking frequency than the NON group, which may be another cause of increased TG and sdLDL-C levels in smokers. However, our multivariate analyses suggest that smoking has an independent effect on these variables as well. In men, values of sdLDL-C/LDL-C ratio were also lower in EX1 than in CUS but were still higher than those in the NON group. Oxidative stress in ex-smokers might be associated with production of sdLDL-C. In addition, hepatic lipase activity has been reported to be higher in smokers than in non-smokers ${ }^{29,30)}$. These data were consistent with our results. Although no supporting data are available, we could set up a hypothesis that hepatic lipase activity in ex-smokers may keep high level after smoking cessation, and further studies should be done in the future.

Our data also indicate that there are gender differences in TG and sdLDL-C changes due to smoking cessation. In men, TG and sdLDL-C were significantly higher in the EX1 group than in the NON group, but in women such differences were not observed. Also, with regard to other lipids parameters, the results in EX1 men were similar to those in EX2 men, while in women, those in EX1 were similar to those in NON. Such differences may be due to the fact that women might use fewer cigarettes for less time, resulting in such gender differences ${ }^{31)}$. In addition, our sample size of EX2 women was smaller than of other groups.

To our knowledge, this is the first study to examine the direct relationship between lipids, especially sdLDL-C, and smoking in a very large population of over 30,000 subjects. Additionally, we have documented that the effects of smoking on lipids, especially on TG and sdLDL-C values, persist for years after smoking cessation. However, our study has some limitations. Our study was cross-sectional in nature. Another shortcoming was that we did not have an accurate count of the number of cigarettes smoked, the precise number of years of smoking and after smoking cessation, and the exact amount of alcohol consumption. Moreover, since the data were collected in only one region of Japan, the results may differ in other regions of Japan and in other ethnic groups. Another potential shortcoming is that our data on smoking and drinking habits were based on selfreported questionnaires, and therefore, subjects may have underreported these habits. In addition, ex- 
smokers may have quit smoking because of illness, and therefore may not have participated in the medical examination and be under-represented in our data base.

In summary, our data clearly shows that current cigarette smoking is associated with elevated levels of TG and sdLDL-C, as well as decreased levels of HDLC. Our results also indicate that smoking effects may persist for a number of years after smoking cessation.

\section{Conclusion}

In conclusion, our data are consistent with the concept that current and prior cigarette smoking is associated with dyslipidemia consisting of elevated TG, sdLDL-C, and decreased HDL-C. Furthermore, elevated TG and sdLDL-C levels remain in ex-smokers in men. Therefore, smoking not only causes endothelial dysfunction, but also promotes dyslipidemia, providing a further reason to promote smoking cessation.

\section{Acknowledgements and Notice of Grant Support}

The contribution of all subjects in this study is gratefully acknowledged and gratefully appreciated.

This study was supported by the university research fund (No.32782) and the research grant (No.2A232) from Denka Co., Ltd., Tokyo, Japan to AM, and presented in part at the 51st Annual Scientific Meeting of the Japan Atherosclerosis Society, July 11 th, 2019 in Kyoto, Japan.

\section{Conflict of Interests}

YI is an employee of Denka Co., Ltd, MA is supported by a research grant from Denka Co., Ltd., Tokyo, Japan. All other authors declare no potential conflicts of interest with respect to the research, authorship, and/or publication of this article.

\section{Author Contributions}

$\mathrm{MN}$ and MA designed and conducted the study. YY, WI, TK and RT recruited study subjects and collected data. MN and MA analyzed the data and EK supervised the analysis. MN and MA wrote the first manuscript and EJS revised it. All authors reviewed and edited the manuscript and approved the final version of the manuscript.

\section{References}

1) WHO, Tobacco [updated 26 July 2019]. https://www.who.int/news-room/fact-sheets/detail/ tobacco (accessed 2 March 2020)

2) Kinoshita M, Yokote $K$, Arai $H$, Iida M, Ishigaki $Y$, Ishibashi S, Umemoto S, Egusa G, Ohmura H, Okamura T, Kihara S, Koba S, Saito I, Shoji T, Daida H, Tsukamoto K, Deguchi J, Dohi S, Dobashi K, Hamaguchi H, Hara M, Hiro T, Biro S, Fujioka Y, Maruyama C, Miyamoto Y, Murakami Y, Yokode M, Yoshida H, Rakugi H, Wakatsuki A, Yamashita S: Japan Atherosclerosis Society (JAS) Guidelines for prevention of Atherosclerosis Cardiovascular Diseases 2017. J Atheroscler Thromb, 2018; 25: 846984

3) Nakai M, Watanabe M, Kokubo Y, Nishimura K, Higashiyama A, Takegami M, Nakao M Y, Okumura T, Miyamoto Y: Development of a cardiovascular disease risk prediction model using the Suita Study, a populationbased prospective cohort study in Japan. J Atheroscler Thoromb, 2020; 27: 1160-1175

4) Lee P N, Fry J S, Hamling J S: Using the negative exponential distribution to quantitatively review the evidence on how rapidly the excess risk of ischaemic heart disease declines following quitting smoking. Regul Toxicol Pharmacol, 2012; 64: 51-67

5) Lloyd-Jones D M, Huffman M D, Karmali K N, Sanghavi D, Wright J S, Pelser C, Gulati M, Masoudi F A, Goff D C Jr: Estimating Longitudinal Risks and Benefits From Cardiovascular Preventive Therapies Among Medicare Patients: The Million Hearts Longitudinal ASCVD Risk Assessment Tool: A Special Report From the American Heart Association and American College of Cardiology. Circulation, 2017; 135: e793-e813

6) Duncan M S, Freiberg M S, Greevy R A Jr, Kundu S, Vasan R S, Tindle H A: Association of Smoking Cessation With Subsequent Risk of Cardiovascular Disease. JAMA, 2019; 322: 642-650

7) Libby P, Loscalzo J, Ridker PM, Farkouh ME, Hsue PY, Fuster V, Hasan AA, Amar S: Inflammation, Immunity, and Infection in Atherothrombosis: JACC Review Topic of the Week. J Am Coll Cardiol, 2018; 72: 2071-2081

8) Golbidi S, Edvinsson L, Laher I: Smoking and Endothelial Dysfunction. Curr Vasc Pharmacol, 2020; 18: 1-11

9) Messner B, Bernhard D: Smoking and cardiovascular disease: mechanisms of endothelial dysfunction and early atherogenesis. Arterioscl Throm Vas, 2014; 34: 509-515

10) Jain RB, Ducatman A: Associations between smoking and lipid/lipoprotein concentrations among US adults aged $>/=20$ years. J Circ Biomark, 2018; 7: 1-10

11) Kar D, Gillies C, Zaccardi F, Webb D, Seidu S, Tesfaye S, Davies M, Khunti K: Relationship of cardiometabolic parameters in non-smokers, current smokers, and quitters in diabetes: a systematic review and meta-analysis. Cardiovasc Diabetol, 2016; 15: 158

12) Gepner AD, Piper ME, Johnson HM, Fiore MC, Baker TB, Stein JH: Effects of smoking and smoking cessation on lipids and lipoproteins: outcomes from a randomized clinical trial. Am Heart J, 2011; 161: 145-151

13) Zhao X, Zhang HW, Zhang Y, Li S, Xu RX, Sun J, Zhu CG, Wu NQ, Gao Y, Guo YL, Liu G, Dong Q, Li JJ: 
Impact of Smoking Status on Lipoprotein Subfractions: Data from an Untreated Chinese Cohort. Biomed Environ Sci, 2017; 30: 235-243

14) Parlesak A, Eckoldt J, Winkler K, Bode CJ, Schafer C: Intercorrelations of lipoprotein subfractions and their covariation with lifestyle factors in healthy men. J Clin Biochem Nutr, 2014; 54: 174-180

15) Otokozawa S, Ai M, Asztalos BF, White CC, DemissieBanjaw S, Cupples LA, Nakajima K, Wilson PW, Schaefer EJ: Direct assessment of plasma low density lipoprotein and high density lipoprotein cholesterol levels and coronary heart disease: results from the Framingham Offspring Study. Atherosclerosis, 2010; 213: 251-255

16) Hirano T, Ito Y, Saegusa H, Yoshino G: A novel and simple method for quantification of small, dense LDL. J Lipid Res, 2003; 44: 2193-2201

17) Ito $Y$, Fujimura M, Ohta M, Hirano T: Development of a homogeneous assay for measurement of small dense LDL cholesterol. Clin Chem, 2011; 57: 57-65

18) Ai M, Otokozawa S, Asztalos BF, Ito Y, Nakajima K, White CC, Cupples LA, Wilson PW, Schaefer EJ: Small dense LDL cholesterol and coronary heart disease: results from the Framingham Offspring Study. Clin Chem, 2010; 56: 967-976

19) Ai M, Otokozawa S, Asztalos BF, Nakajima K, Stein E, Jones PH, Schaefer EJ: Effects of maximal doses of atorvastatin versus rosuvastatin on small dense low-density lipoprotein cholesterol levels. Am J Cardiol, 2008; 101: 315-318

20) Ikezaki H, Ai M, Schaefer EJ, Otokozawa S, Asztalos BF, Nakajima K, Zhou Y, Liu CT, Jacques PF, Cupples LA, Furusyo N: Cardiovascular disease prevalence and insulin resistance in the Kyushu-Okinawa Population Study and the Framingham Offspring Study. J Clin Lipidol, 2017; 11: 348-356

21) Hsu H, Hsu P, Ming-Hui C, Ito Y, Kanda E, Schaefer EJ, Ai M: Lipoprotein Subfractions in Prediabetes and Diabetes in Taiwan. J Atheroscler Thromb, 2019; 26: 890-914

22) Friedewald WT, Levy RI, Fredrickson DS: Estimation of the concentration of low-density lipoprotein cholesterol in plasma, without use of the preparative ultracentrifuge. Clin chem, 1972; 18: 499-502

23) The Japanese Society of Hypertension: The Japanese Society of Hypertension Guidelines for the Management of Hypertension 2019 (JSH2019), Lifescience Co., Ltd., Tokyo, Japan, 2019

24) Chelland Campbell S, Moffatt RJ, Stamford BA: Smoking and smoking cessation -- the relationship between cardiovascular disease and lipoprotein metabolism: a review. Atherosclerosis, 2008; 201: 225-235

25) Chajek-Shaul T, Berry EM, Ziv E, Friedman G, Stein O, Scherer G, Stein Y: Smoking depresses adipose lipoprotein lipase response to oral glucose. Eur J Clin Invest, 1990; 20: 299-304

26) McNamara JR, Jenner JL, Li Z, Wilson PW, Schaefer EJ: Change in LDL particle size is associated with change in plasma triglyceride concentration. Arterioscler Thromb, 1992; 12: 1284-1290

27) Austin MA, Breslow JL, Hennekens CH, Buring JE, Willett WC, Krauss RM: Low-density lipoprotein subclass patterns and risk of myocardial infarction. JAMA, 1988; 260: 1917-1921

28) Campos H, Genest JJ Jr, Blijlevens E, McNamara JR, Jenner JL, Ordovas JM, Wilson PW, Schaefer EJ: Low density lipoprotein particle size and coronary artery disease. Arterioscle Thromb, 1992; 12: 187-195

29) Eliasson B, Mero N, Taskinen MR, Smith U: The insulin resistance syndrome and postprandial lipid intolerance in smokers. Atherosclerosis, 1997; 129: 79-88

30) Kong C, Nimmo L, Elatrozy T, Anyaoku V, Hughes C, Robinson S, Richmond W, Elkeles RS: Smoking is associated with increased hepatic lipase activity, insulin resistance, dyslipidaemia and early atherosclerosis in Type 2 diabetes. Atherosclerosis, 2001; 156: 373-378

31) National Institute of Health and Nutrition Japan, The National Health and Nutrition Survey Japan, 2017. https://www.mhlw.go.jp/stf/seisakunitsuite/bunya/ kenkou_iryou/kenkou/eiyou/h29-houkoku.html (accessed 2 March 2020) 